\title{
Degradabilidade ruminal da fibra das frações do resíduo industrial de tomate
}

\author{
[Ruminal degradability of fiber of tomato industrial residues fractions]
}

\author{
W.E. Campos ${ }^{1}$, A.L.C.C. Borges ${ }^{2 *}$, H.M. Saturnino ${ }^{2}$, R.R. Silva ${ }^{3}$, B.M. Sousa ${ }^{1}$ \\ M.C.P. Rogério ${ }^{4}$, I.Borges ${ }^{3}$, N.M. Rodríguez ${ }^{3}$ \\ ${ }^{1}$ Aluno de pós-graduação - EV-UFMG \\ ${ }^{2}$ Escola de Veterinária - UFMG \\ Caixa Postal 567 \\ 30123-970 - Belo Horizonte, MG \\ ${ }^{3}$ Zootecnista autônomo \\ ${ }^{4}$ Universidade Estadual Vale do Acaraú - CE
}

\begin{abstract}
RESUMO
Avaliou-se a degradabilidade in situ da fibra em detergente neutro (FDN) e da fibra em detergente ácido (FDA) das cascas e das sementes inteiras ou moídas (peneira de $2 \mathrm{~mm}$ ) do resíduo industrial de tomate (RIT). Foram utilizados três bovinos adultos, machos, em delineamento de blocos ao acaso com parcelas subdivididas. A solubilidade da FDN apresentou baixos valores para todas as frações estudadas, sendo semelhante entre cascas $(3 \%)$, sementes inteiras (5\%) e resíduo (7\%). Sementes moídas apresentaram maior solubilidade da FDN (20\%). Os potenciais de degradação da FDN para sementes moídas, RIT, cascas e sementes inteiras foram de 78, 64, 36 e 33\%, respectivamente. As taxas de degradação da FDN variaram de 4,4 a $14,8 \%$ /h para as diferentes frações do RIT, sendo de $9 \% / \mathrm{h}$ para o resíduo integral. Os parâmetros de degradação da FDA seguiram a mesma tendência da FDN. Concluiu-se que, apesar de a FDN e a FDA do RIT terem apresentado altas taxas de degradação, o potencial de degradação dessas frações depende do processamento, pois sementes inteiras apresentaram degradabilidades muito inferiores às das sementes moídas.
\end{abstract}

Palavras-chave: tomate, in situ, casca, semente, FDN, FDA

\begin{abstract}
The in situ procedure was used to evaluate the disappearance of neutral detergent fiber (NDF) and acid detergent fiber $(A D F)$ of skins and whole or grounded (sieve of $2 \mathrm{~mm}$ ) seeds of tomato by-product (TBP). Three steers were used in a split-plot block design. The solubility of NDF showed low values for all the evaluated fractions, been similar among skins (3\%), whole seeds (5\%) and total TBP (7\%). Grounded seeds showed higher NDF solubility (20\%). The NDF potential degradability for grounded seeds, TBP, skins and whole seed were 78, 64, 63 and 33\%, respectively. NDF degradability rates varied from 4.4 to $14.8 \% / h$ for all TBP fractions, been $9 \% / h$ for total TBP. The ADF parameters followed the same trend as NDF. TBP NDF and ADF showed good degradability rates, but the potential degradability of these fractions depends on the showed processing, since whole seeds NDF and ADF showed degradability much lower than grounded seeds.
\end{abstract}

Keywords: tomato, in situ, skin, seed, NDF, ADF

Recebido em 24 de novembro de 2005

Aceito em 18 de setembro de 2006

*Autor para correspondência (corresponding author)

E-mail: anaglaub@vet.ufmg.br

Apoio: CNPQ e Equipe Prodap 


\section{INTRODUÇÃO}

A efetividade da fibra pode ser definida como a habilidade de estimular a mastigação e/ou a habilidade de manter a gordura do leite, sendo a FDN efetiva definida como a fração do alimento que estimula a atividade mastigatória e o grau de efetividade determinado pela capacidade de estimulação mastigatória (Grant, 1997).

O NRC (Nutrient..., 2001) recomenda no mínimo $25 \%$ de FDN na dieta, devendo $76 \%$ desse serem supridos por forragens grosseiras, podendo o restante ser suprido por fontes de fibra não forrageiras (FFNF). Para dietas pobres em fibra, a concentração de FDN das forragens e especialmente o tamanho de partículas dessas tornam-se essenciais para o estímulo da ruminação e para o aproveitamento das FFNF. Isso porque a competição entre a degradação ruminal e a taxa de passagem pelo rúmen são importantes fatores que determinam a utilização desses alimentos (Faichney, 1984; Grant, 1997).

Devido ao elevado teor de fibra, o resíduo industrial de tomate pode ser classificado como uma FFNF, entretanto esse alimento possui alta proporção de proteína e lipídeos em relação às
FFNF comumente encontradas no país, o que torna sua utilização mais desejável. Portanto, o conhecimento da dinâmica de fermentação da fração fibrosa desse alimento torna-se imprescindível para sua correta utilização na alimentação de ruminantes. $\mathrm{O}$ objetivo deste experimento foi avaliar a cinética de degradação ruminal da fibra em detergente neutro e fibra em detergente ácido das diferentes frações que compõem o RIT.

\section{MATERIAL E MÉTODOS}

Avaliou-se a degradabilidade in situ do resíduo industrial de tomate e de suas diferentes frações, casca e sementes. Foram utilizados três bovinos adultos, machos, da raça Nelore, fistulados no rúmen e alimentados em cochos individualizados, tendo livre acesso à comida $\mathrm{e}$ água. A dieta, com $14 \%$ de proteína bruta na matéria seca, composta de silagem de milho $(17 \%)$, resíduo de tomate $(30 \%)$, caroço de algodão $(2,9 \%)$, sorgo $(44,3 \%)$, soja $(3,8 \%)$ e minerais $(2 \%)$, foi fornecida às $7 \mathrm{~h}$ e $17 \mathrm{~h}$, de forma a permitir $10 \%$ de sobras. A composição desses alimentos é mostrada na Tab. 1.

Tabela 1. Composição bromatológica do resíduo industrial de tomate (RIT), silagem de milho (SM), caroço de algodão (CA), sorgo grão (SG) e farelo de soja (FS) que compuseram a dieta dos animais, em porcentagem da MS

\begin{tabular}{lrrrrrrc} 
& MS & PB & FDN & FDA & EE & Lignina & $\begin{array}{c}\% \text { NIDA } \\
\text { no N-total }\end{array}$ \\
\hline RIT & 22,1 & 20,5 & 63,1 & 50,8 & 14,9 & 17,9 & 18,6 \\
SM & 45,1 & 7,5 & 52,3 & 25,7 & 4,1 & 3,5 & - \\
CA & 91,1 & 23,2 & 46,7 & 34,5 & 20,7 & 14,5 & 3,6 \\
SG & 88,2 & 11,0 & 14,5 & 7,7 & 3,7 & 0,3 & 13,2 \\
FS & 91,4 & 51,1 & 27,1 & 12,4 & 2,1 & 0,4 & 1,3 \\
\hline
\end{tabular}

$\mathrm{MS}=$ matéria seca, $\mathrm{PB}=$ proteína bruta, $\mathrm{FDN}=$ fibra em detergente neutro, $\mathrm{FDA}=$ fibra em detergente ácido e NIDA = nitrogênio insolúvel em detergente ácido.

Foram incubados, em sacos de náilon de $7 \mathrm{x}$ $13 \mathrm{~cm}$ com poros de 50 micras ancorados em uma corrente, $5 \mathrm{~g}$ de RIT, cascas e sementes de tomate, sendo a composição dos mesmos demonstrada na Tab. 2. Os dois primeiros alimentos foram moídos em peneira de $2 \mathrm{~mm}$, e as sementes foram incubadas inteiras ou moídas a $2 \mathrm{~mm}$. Os tempos de incubação foram $0,3,8$, $16,24,48$ e 72 horas, sendo utilizados sacos vazios em cada um dos tempos para se ajustar a contaminação da malha (Mertens, 1993).

Os saquinhos com as amostras, quatro para cada amostra e tempo, foram sempre colocados após o fornecimento da primeira refeição (7h) e retirados nos tempos citados anteriormente, sendo, então, lavados manualmente em água corrente. Após os tempos preestabelecidos, foram secos em estufa ventilada a $60^{\circ} \mathrm{C}$ e 
pesados. Determinaram-se as frações desaparecidas de FDN e FDA (Van Soest et al., 1991). Para a caracterização do material incubado, determinou-se o teor de proteína bruta, FDN, FDA, extrato etéreo, lignina e nitrogênio insolúvel em detergente ácido nas amostras originais (Official..., 1980; Van Soest et al., 1991).

Para avaliação do ambiente ruminal, foram coletadas amostras de líquido ruminal à 1 , às 3 , $6,9,12,15,18,21$ e $24 \mathrm{~h}$ após a alimentação, sendo determinadas as concentrações de $\mathrm{N}-\mathrm{NH}_{3}$.

A comparação das médias e os coeficientes do modelo proposto por Orskov e McDonald (1979) foram determinados utilizando-se o programa SAEG 8.0 (Sistema ..., 1997). Após a determinação dos parâmetros do modelo, estimou-se a degradabilidade efetiva adotando-se as taxas de passagem de 2,5 e $8 \% /$ h (McDonald, 1981).

O experimento seguiu um delineamento de blocos (animais) ao acaso com parcelas subdivididas (tempos de incubação), utilizandose, para avaliar as diferenças entre as médias obtidas, o teste SNK $(\mathrm{P}<0,05)$, comparando-se os valores dos diferentes tempos de incubação para cada fração.

\section{RESULTADOS E DISCUSSÃO}

A composição bromatológica do RIT e das frações incubadas se encontra na Tab. 2.

Tabela 2. Composição bromatológica das cascas de tomate (RIT-C), do resíduo industrial de tomate integral (RIT) e das sementes de tomate (RIT-S) incubados, em porcentagem da MS

\begin{tabular}{lcccccc}
\hline & PB & FDN & FDA & EE & Lignina & $\begin{array}{c}\text { \%NIDA no } \\
\text { N-Total }\end{array}$ \\
\hline RIT-C & 10,8 & 62,5 & 52,4 & 3,6 & 20,4 & 22,0 \\
RIT & 21,8 & 61,7 & 38,8 & 15,5 & 17,3 & 18,6 \\
RIT-S & 29,0 & 59,9 & 29,9 & 22,4 & 16,9 & 18,6 \\
\hline
\end{tabular}

$\mathrm{PB}=$ proteína bruta, $\mathrm{FDN}=$ fibra em detergente neutro, $\mathrm{FDA}=$ fibra em detergente ácido, $\mathrm{EE}=$ extrato etéreo, $\mathrm{NIDA}=$ nitrogênio insolúvel em detergente ácido.

A maior proporção da lignina encontra-se nas cascas, entretanto as sementes também possuem consideráveis concentrações desse composto. Outra diferença na composição das sementes e das cascas são os teores de celulose, 13 e $32 \%$, e hemicelulose, 30 e 10,1\%, respectivamente.

Ao utilizarem a técnica do ácido sulfúrico com ou sem a prévia avaliação da FDN para avaliar a concentração de lignina no resíduo de tomate, Weiss et al. (1997) obtiveram resultados similares, o que indica a ausência de taninos no resíduo, pois o detergente neutro é responsável pela remoção desses compostos (Van Soest et al., 1991).

Os desaparecimentos médios da FDN e FDA estão demonstrados, respectivamente, nas Tab. 3 e 4 .
A solubilidade da FDN apresentou valores inferiores a 20 para todos os alimentos estudados, sendo semelhante entre cascas, sementes inteiras e o resíduo. Apenas as sementes moídas apresentaram maior solubilidade da FDN, podendo tal fato ser justificado pela perda de material pelos poros dos saquinhos. Isso é reforçado pelo fato de Machado et al. (1996) terem verificado valores próximos de zero para a solubilidade da FDN do RIT.

Desconsiderando as três primeiras horas de incubação, as quais são mais influenciadas pela fração solúvel do alimento, verificou-se que o desaparecimento da FDN diferiu em todos os alimentos, obedecendo à seguinte ordem decrescente de degradação: sementes moídas, RIT, cascas de tomate e sementes inteiras. Dessa forma, observou-se que as sementes, dependendo do processamento, podem ser muito $(79 \%)$ ou pouco aproveitadas $(32 \%)$ pelo animal. 
Tabela 3. Desaparecimento médio (\%) da fibra em detergente neutro das cascas de tomate (RIT-C), resíduo industrial de tomate (RIT), sementes de tomate moídas (RIT-S 2mm) e inteiras (RIT-SI) no tempo zero e em diferentes tempos após a incubação ruminal

\begin{tabular}{ccccc}
\hline Tempo & RIT-C & RIT & RIT-S 2mm & RIT-SI \\
\hline 0 & $3,1 \mathrm{eB}$ & $7,0 \mathrm{~dB}$ & $19,9 \mathrm{eA}$ & $4,8 \mathrm{fB}$ \\
3 & $9,9 \mathrm{dC}$ & $27,0 \mathrm{cB}$ & $40,1 \mathrm{dA}$ & $9,5 \mathrm{eC}$ \\
8 & $23,4 \mathrm{cC}$ & $50,8 \mathrm{bB}$ & $63,1 \mathrm{cA}$ & $14,3 \mathrm{dD}$ \\
16 & $28,4 \mathrm{bC}$ & $55,5 \mathrm{aB}$ & $69,3 \mathrm{bA}$ & $18,4 \mathrm{cD}$ \\
24 & $31,4 \mathrm{bC}$ & $57,4 \mathrm{aB}$ & $75,1 \mathrm{aA}$ & $24,3 \mathrm{bD}$ \\
48 & $35,4 \mathrm{aC}$ & $59,9 \mathrm{aB}$ & $79,3 \mathrm{aA}$ & $29,4 \mathrm{aD}$ \\
72 & $37,9 \mathrm{C}$ & $60,3 \mathrm{aB}$ & $79,2 \mathrm{aA}$ & $32,5 \mathrm{aD}$ \\
\hline
\end{tabular}

Valores seguidos de letras minúsculas diferentes na coluna ou letras maiúsculas na linha diferem entre si $(\mathrm{P}<0,05)$ pelo teste SNK.

Tabela 4. Desaparecimento médio (\%) da fibra em detergente ácido das cascas de tomate (RIT-C), resíduo industrial de tomate (RIT), sementes de tomate moídas (RIT-S 2mm) e inteiras (RIT-SI) no tempo zero e em diferentes tempos após a incubação ruminal

\begin{tabular}{ccccc}
\hline Tempo & RIT-C & RIT & RIT-S 2mm & RIT-SI \\
\hline 0 & $0,1 \mathrm{eC}$ & $6,2 \mathrm{~dB}$ & $30,1 \mathrm{eA}$ & $5,2 \mathrm{eB}$ \\
3 & $4,6 \mathrm{eD}$ & $19,2 \mathrm{cB}$ & $48,7 \mathrm{dA}$ & $13,2 \mathrm{dC}$ \\
8 & $17,6 \mathrm{dC}$ & $40,6 \mathrm{bB}$ & $67,7 \mathrm{cA}$ & $21,3 \mathrm{cC}$ \\
16 & $21,6 \mathrm{cdD}$ & $45,8 \mathrm{abB}$ & $71,0 \mathrm{bcA}$ & $27,3 \mathrm{bC}$ \\
24 & $24,9 \mathrm{bcD}$ & $45,5 \mathrm{abB}$ & $75,0 \mathrm{abA}$ & $33,2 \mathrm{aC}$ \\
48 & $28,8 \mathrm{abD}$ & $49,4 \mathrm{aB}$ & $77,8 \mathrm{aA}$ & $37,3 \mathrm{aC}$ \\
72 & $31,6 \mathrm{aD}$ & $49,4 \mathrm{aB}$ & $77,7 \mathrm{aA}$ & $38,6 \mathrm{aC}$ \\
\hline
\end{tabular}

Valores seguidos de letras minúsculas diferentes na coluna ou letras maiúsculas na linha diferem entre si $(\mathrm{P}<0,05)$ pelo teste SNK.

As taxas de degradação da FDN variaram de 4,4 a $14,8 \% / \mathrm{h}$ (Tab. 4) para os diferentes alimentos, sendo de $8,96 \% / \mathrm{h}$ para o resíduo integral. Esse valor é inferior ao verificado por Machado et al. (1996), que relataram taxa de degradação do RIT de 11 a $13 \% / \mathrm{h}$ para animais que receberam de zero a $80 \%$ de RIT na dieta. Entretanto, os autores avaliaram a degradação do RIT por $24 \mathrm{~h}$, o que pode resultar em valores superestimados, uma vez que a fermentação da FDN pode se estabilizar após esse período, como verificado para as cascas (Tab.3).

Altas taxas de degradação, como as relatadas anteriormente, podem ser atribuídas à grande superfície de contato do RIT, uma vez que a espessura das cascas é bastante reduzida.

Considerando os alimentos moídos, verificou-se que as sementes e o RIT apresentaram 78 e 59,4\%, respectivamente, de degradação potencial. Entretanto, se consideradas as sementes inteiras e as cascas, obteve-se valor médio (33,3 e 36,4\%, respectivamente) de degradabilidade potencial muito inferior ao RIT, demonstrando que, caso a ruminação não seja efetiva em romper a estrutura da semente, a digestibilidade total do RIT pode ficar comprometida. Ao avaliarem o desaparecimento ruminal da FDN do fruto do tomate, Cerda et al. (1995) verificaram $14 \%$ de degradação, valor muito inferior ao verificado na Tab. 5. Isso pode ser devido à diferença de composição do subproduto.

A diferença de solubilidade da FDA para as frações do RIT estudadas foi maior do que da FDN, sendo o desaparecimento maior para as sementes moídas. Com o intuito de reduzir as perdas de MS pelos poros dos saquinhos, alguns pesquisadores (Barrell et al., 2000; Burke et al., 2000) sugeriram que o material seja apenas picado de forma a se obter partículas de tamanhos semelhantes às partículas ruminadas. 
Tabela 5. Parâmetros de degradação ruminal da fibra em detergente neutro das cascas de tomate (RIT-C), resíduo industrial de tomate (RIT), sementes de tomate moídas (RIT-S 2mm) e inteiras (RIT-SI)

\begin{tabular}{ccccc}
\hline Parâmetro & RIT-C & RIT & RIT-S 2mm & RIT-SI \\
\hline A+B & 36,4 & 59,4 & 78 & 33,3 \\
A (\%) & 3 & 6,2 & 20 & 5,4 \\
B (\%) & 33,4 & 53,2 & 58 & 27,9 \\
C (\%/h) & 9,58 & 8,96 & 14,77 & 4,4 \\
r $^{2}$ & 0,96 & 0,98 & 0,98 & 0,93 \\
\hline
\end{tabular}

$\mathrm{A}+\mathrm{B}=$ potencial de degradação, $\mathrm{A}=$ fração solúvel, $\mathrm{B}=$ fração insolúvel potencialmente degradável, $\mathrm{C}=$ taxas de degradação, $\mathrm{r}^{2}=$ coeficiente de determinação.

Verificou-se que a estabilização dos desaparecimentos da FDA do RIT, das sementes e das cascas se deram, respectivamente, às 16,24 e $48 \mathrm{~h}$ de incubação. Além de se estabilizar tardiamente, as cascas também apresentaram baixo potencial de degradação da FDA, sendo esse decorrente da fração solúvel que foi praticamente nula, associado ao pequeno valor da fração insolúvel potencialmente degradável (B), como observado na Tab. 6.

Tabela 6. Parâmetros de degradação ruminal da fibra em detergente ácido das cascas de tomate (RIT-C), resíduo industrial de tomate (RIT), sementes de tomate moídas (RIT-S 2mm) e inteiras (RIT-SI)

\begin{tabular}{ccccc}
\hline Parâmetro & RIT-C & RIT & RIT-S 2mm & RIT-SI \\
\hline A+B & 30,2 & 48,7 & 76,6 & 38,4 \\
A (\%) & 0 & 5,1 & 30 & 5,9 \\
B (\%) & 30,2 & 43,7 & 46,6 & 32,5 \\
C (\%/h) & 8,44 & 16,76 & 17,98 & 7,46 \\
r $^{2}$ & 0,94 & 0,96 & 0,96 & 0,93 \\
\hline
\end{tabular}

$\overline{\mathrm{A}+\mathrm{B}=\text { potencial de degradação, } \mathrm{A}=\text { fração solúvel, } \mathrm{B}=\text { fração insolúvel potencialmente degradável, } \mathrm{C}=\text { taxas de }}$ degradação, $\mathrm{r}^{2}=$ coeficiente de determinação.

Os parâmetros de degradação da FDA seguiram a mesma tendência da FDN, sendo o potencial de degradação das cascas e das sementes inteiras bastante baixos, apesar da alta taxa de degradação. Tal fato pode ser atribuído ao efeito físico da lignina que se distribui pelos tecidos das plantas, impedindo a degradação da celulose e hemicelulose. Essa interação planta/animal interfere na degradação (Akin, 1989) e na passagem do alimento, o que pode afetar o consumo (Forbes, 1995).

É possível que diferenças na degradação de paredes celulares estejam relacionadas às ligações químicas e físicas que unem seus três componentes principais: celulose, hemicelulose e lignina. A hemicelulose parece estar mais intimamente associada à lignina que a celulose, o que justifica a diminuição mais acentuada da digestão da hemicelulose do que da celulose com o avanço da maturidade da planta (Thiago e Gill, 1993). O mecanismo de ação da lignina parece envolver um efeito físico por formação de uma barreira sobre a hemicelulose e celulose (teoria da incrustação) e também por prevenir a adesão dos microrganismos à parede celular (Thiago e Gill, 1993).

$\mathrm{Na}$ Tab. 7 verifica-se que a degradabilidade efetiva das frações fibrosas apresentou valores inferiores a 50\%, exceto para as sementes moídas. Tal fato deveu-se às baixas solubilidades associadas às baixas taxas de degradação das frações fibrosas indicadas nas Tab. 5 e 6 . 
Tabela 7. Degradabilidades efetivas (\%) da fibra em detergente neutro (FDN) e fibra em detergente ácido (FDA) das cascas (RIT-C), resíduo industrial de tomate integral (RIT-I), sementes de tomate moídas (RIT-S2mm) e inteiras (RIT-SI) de acordo com a taxa de passagem

\begin{tabular}{lccc}
\multirow{2}{*}{\multicolumn{1}{c}{ Nutriente/Alimento }} & \multicolumn{3}{c}{ Taxa de passagem } \\
\cline { 2 - 4 } & $0,02 / \mathrm{h}$ & $0,05 / \mathrm{h}$ & $0,08 / \mathrm{h}$ \\
\hline FDN / RIT-C* & 30,6 & 24,9 & 21,2 \\
FDN / RIT-I* & 49,7 & 40,4 & 34,3 \\
FDN / RIT-S2mm* & 71,1 & 63,4 & 57,7 \\
FDN / RIT-SI & 24,6 & 18,5 & 15,3 \\
FDA / RIT-C* & 24,4 & 18,9 & 15,5 \\
FDA / RIT-I* & 44,1 & 38,7 & 34,6 \\
FDA / RIT-S2mm* & 71,9 & 66,4 & 62,2 \\
FDA / RIT-SI & 31,5 & 25,3 & 21,6 \\
\hline
\end{tabular}

* Material moído em moinho com peneira de $2 \mathrm{~mm}$.

$\mathrm{Na}$ avaliação em conjunto da degradabilidade efetiva da FDN e FDA das sementes, notou-se significativa melhora quando as sementes foram moídas, observando-se aumentos da degradabilidade efetiva de $326 \%$ para a FDN e $259 \%$ para a FDA, considerando-se todas as taxas de pasagem. Tal diferença pode justificar um investimento em máquinas que processem o RIT antes do fornecimento aos animais, pois isso provavelmente resultaria em melhor desempenho animal.

As degradabilidades efetivas da FDN e FDA das cascas e sementes inteiras mostraram que apenas um terço a um quinto do material ingerido é degradado. Tal fato pode evidenciar a possibilidade de grande restrição de consumo em razão da baixa digestibilidade, entretanto, devido ao pequeno tamanho de partícula, tal efeito provavelmente não ocorra, uma vez que adequadas taxas de ganho têm sido observadas em confinamentos de bovinos de corte ao se utilizar até $55 \%$ de RIT na dieta (dados não publicados). Por outro lado, alimentos de baixa digestibilidade tendem a agravar as questões ambientais por aumentar o volume de excreta dos animais.

\section{CONCLUSÕES}

O resíduo industrial de tomate pode constituir boa fonte de nutrientes para os microrganismos ruminais e para o animal.

\section{REFERÊNCIAS BIBLIOGRÁFICAS}

AKIN, D.E. Histological and physical factors affecting digestibility of forages. Agron. J., v.81, p.17-25, 1989.

BARRELL, L.G.; BURKE, J.L.; WAGHORN, G.C. Preparation of fresh forages for incubation and prediction of nutritive value. Proc. N. Zeal. Soc. Anim. Prod., v.60, p.5-8, 2000.

BURKE, J.L.; WAGHORN, G.C.; BROOKES, I.M. Formulating total mixed rations from forages - defining the digestion kinetics of contrasting. Proc. N. Zeal. Soc. Anim. Prod., v.60, p.9-14, 2000.

CERDA, D.A.; MANTEROLA, H.B.; SIRHAN, L.A.Y. et al. Estudio del uso de residuos agoindustriales en alimentacion animal. Estúdio de la disponibilidad y foalo nutritivo de cinco cultivos horticolas en la zona central de Chile. Av. Prod. Anim., v.20, p.191-204, 1995.

FAICHNEY, G.J. Control of digestion and metabolism in ruminants: the kinetics of particulate matter in the rumen. In: INTERNATIONAL SYMPSIUM ON RUMINANT PHYSIOLOGY, 6., 1984. Proceedings... Banff: CAB International, 1984.

FORBES, J.M. Voluntary food intake and diet selection in farm animals. Guildford, UK: Cab International, 1995. 532p.

GRANT, R.J. Interactions among forrages and non forages sources. J. Dairy Sci., v.80, p.14381446, 1997. 
MACHADO, C.; MANTEROLA, H.B.; PORTE, E.F. et al. Efecto del nivel de inclusión de pomaza de tomate sobre la degradabilidad de la materia seca, proteína y FDN de los componentes dietarios. Av. Prod. Anim., v.21, p.115-124, 1996.

McDONALD, I. A revised model for the estimation of protein degradability in the rumen. J. Agric. Sci., v.96, p.251-252, 1981.

MERTENS, D.R. Rate and extent of digestion. In: FORBES, J.M.; FRANCE, A.M. Quantitative aspects of ruminant digestion and metabolism. J. Northampton: CAB International, 1993. cap.2, p.13-52.

NUTRIENT requirements of dairy cattle. 7.ed. Washington, DC: National Academy, 2001. 380p.

OFICCIAL Methods of Analysis. 13. ed. Washington, DC: AOAC, 1980. 1015p.

ORSKOV, E.R.; McDONALD, J. The estimation of protein degradability in the rumen from incubation measurements weighted according to rate of passage. J. Agric. Sci., v.92, p.499-503, 1979.

SISTEMA de análises estatísticos e genéticos SAEG. Viçosa:UFV, 1997. 52p.

THIAGO, L.R.L.; GILL, M. Consumo voluntário: fatores relacionados com a degradação e passagem da forragem pelo rúmen. Documentos EMBRAPA - CNPGC, n.43, p.1-65p.1993.

VAN SOEST, P.J.; ROBERTSON, JB.; LEWIS, B.A. Methods for dietary fiber, neutral detergent fiber, and nonstarch polysaccharides in relation to animal nutrition. J. Dairy Sci., v.74, p.35833597, 1991.

WEISS, W. P.; FROBOSE, D. L.; KOCH, M. E. Wet tomato pomace ensiled with corn plants for dairy cows. J. Dairy Sci., v.80, p.2896-2900, 1997. 